\title{
ஆலமர்செல்வனும் ஆடல்வல்லானும்
}

\section{நா. தனலெட்சுமி அ, *, சே. செந்தமிழ்ப்பாவை அ}

அ தமிழ்ப் பண்பாட்டு மையம், அழகப்பா பல்கலைக்கழகம், காரைக்குடி-630004, தமிழ்நாடு, இந்தியா

\section{Alamarcelvan and Atalvallan}

\author{
N. Dhanalakshmi a, *, S. Senthamilppavai a \\ ${ }^{a}$ Centre for Tamil Culture, Alagappa University, Karaikudi - 630004, Tamil Nadu, India.
}

* Corresponding Author: danam.anand@gmail.com

Received: 04-01-2021 Revised: 18-04-2021

Accepted: 24-04-2021

Published: 30-04-2021

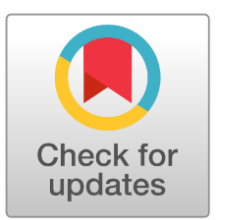

\begin{abstract}
The origin for the divine forms of Lord Dakshinamoorthy also named as south facing deity and Lord Natarajar, which are commonly seen in siva temples are described in Sangam literature. It reveals the importance and antiquity of siva moorthy forms two thousand years ago. The two forms that are incorrectly featured in today's Saiva temples are the Natarajar Alamarselvan and Adalvallan, who sit under a stone tree and teach wisdom. Both forms embody glorious theology. Are of special interest in the Sanskrit literature of over two thousand years ago. Therefore, the purpose of this article is to explain the Alamarselvan and Adalvallan portraits and their philosophy, as pointed out in the Sangam literature.
\end{abstract}

Keywords: Natarajar, Dakshinamoorthy, siva moorthy, Alamarselvan, Adalvallan.

\section{முன்னுரை}

தொல்காப்பியர் சிவனைப் பற்றி யாதும் கூறவில்லை. சங்க நூல்களில் சிவன் என்னும் பெயர் யாண்டும் இடம் பெற்றிலது. எனினும் சிவனுடைய இயல்புகள் பலவும் கூறப்பட்டுள்ளன. தொகை நூல்கட்கு வணக்கச் செய்யுள் பாடிய பெருந்தேவனாருடைய செய்யுளில் சிவனுடைய உருவத் திருமேனி புகழப்பட்டுள்ளது. சிலம்பிலும் மணிமேகலையிலும் கூடச் சிவன் முதன்மை பெறுவது குறிப்பிடத்தக்கதாகும்.

“ஏற்றுவலன் உயரிய எரிமருள் அவிர்சடை

மாற்றரும் கணிச்சி மணிமிடற்றோனும்" (புறநா.56)

எனப் புறநானூற்றில் சிவன் புகழப்பட்டுள்ளான். “நீலமணிமிடற்று ஒருவன்” என ஒளவையார் குறிப்பிட்டுள்ளார் (Puliyur Kesigan, 2010). சிவன் ஐந்து பூதங்களையும் படைத்தவன் என்றும், மழுவாளையுடைய நெடியோன் என்றும் மதுரைக்காஞ்சி புகழ்கின்றது (Somasundaranar, 1980). பெரும் புகழ்மிக்க நவிர மலையில் உறைந்த காரியுண்டிக் கடவுளை (நஞ்சுண்டான்) மலைபடுகடாம் குறிப்பிடுகிறது (Somasundaranar, 1980). திருமுருகாற்றுப்படை சிவபெருமான் வெள்ளேற்றுக் கொடியினை வலத்தில் உயர்த்தியவன், பலரும் புகழும் திண்டோளினன், உமையாளைப் பாகமாகக் கொண்டவன், இமையாத முக்கண்ணை உடையான், மூவெயில் அழித்த முரண்மிகு செல்வன் என்றும் உரைக்கின்றது. ஆல்கெழு கடவுள் (திருமுருகு.256) என்றும் கூறப்பட்டுள்ளான். 
புறநானூற்றில் சிவன் கோயில் “முக்கட் செல்வன் நகர்” எனச் (புறநா.6:18) சுட்டப்படுகிறது. சிவபெருமானின் பிறைநுதல் விளங்கும் கண்ணைப் புறநானூறு 55 ஆம் செய்யுள் சுட்டியுள்ளது. சிவபெருமான் இடைவிடாமல் வேதத்தை மொழிபவன் என்றும் நீண்ட சடையை உடையவன் என்பதை

“நன்றாய்ந்த நீள்நிமிர் சடை முதுமுதல்வன் வாய்போகா

தொன்று புரிந்த ஈரிரண்டின்

ஆறுணர்ந்த ஒருமுது நூல் - புறநா.166

என்று ஆவூர் மூலங்கிழார் குறிப்பிட்டுள்ளார் (Puliyur Kesigan, 2010).

\section{ஆலமர் செல்வன்}

அறனறிந்து மூத்த அறிவுடைய அந்தணர்கள் தமக்கு ஏற்பட்ட ஐயங்களை நீக்குவதற்கு இறைவனிடம் முறையிட அவன் ஆலமரத்தின் கீழிருந்து அவ்ஐயங்களை நீக்கி அருளியதோடு, அவர்கள் மேலும் உணரத்தக்க அறங்களையும் அருளினான் என்பது வரலாறு. இவ்வாறு அறமுணர்த்தும் குருமூர்த்தியின் வடிவே ஆலமர்செல்வன் ஆகும். இவ்ஆலமர்செல்வன் தென்முகக் கடவுள் என்றும் தட்சிணாமூர்த்தி என்றும் அழைக்கப்பெறுகின்றார்.

'ஆலமர் கடவுளன்ன நின் செல்வம்'

'ஆல்கெழு கடவுட் புதல்வ'

'ஆலமர் செல்வன்'

'ஆலமர் செல்வதற்கு அமர்ந்தனன் கொடுத்த'

என்ற பாடல் வரிகளின் மூலம் இக்குருவடிவம் குறிப்பிடப்பட்டுள்ளன (Puliyur Kesigan, 2010; Somasundaranar, 1980).

'அருந்தவருக்கு ஆலின்கீழ் அறமுதலா நான்கனையும்

இருந்தவர்க்கு அருளுமது எனக்கறிய இயம்பேடி

அருந்தவருக்கு அறமுதல் நான்கு என்றருளிச் செய்திலனேல்

திருந்தவருக்கு உலகியற்கை தெரியாகாண் சாழலோ'

என்ற பாடல் இறைவன் ஆலின் கீழிருந்து அறமுரைத்தலின் பயனை விளக்குகின்றது.

\section{ஆடல்வல்லான் - ஸ்ரீ நடராஜ கோலத்தின் ரகசியம்}

பிரஹ்மபுரமாகிய சிதம்பரத்தில் (சிதாகாசத்தின்கண்) கண்ணுதற்பெருமான் நடராஜ கோலமாகலிருந்தாடுவது "அப்பெருமான் அசைந்தாலன்றி அணுவும் அசையாது; அப்பெருமான் ஆட்ட உயிருள்ளபொருள் உயிரில்லாத பொருளென்னு மிரண்டுமாடுகின்றன" என்பதை விளக்குவதாம். இதுபற்றித் தான் பெரியார் "ஆட்டுவித்தா லாரொருவராடாதாரே" என்று திருவாய் மலர்ந்தருளினர். இந்த நடராஜகோலம் "உயிர்கட்கு இயல்பாக ஒரு சுதந்திரமுமில்லை. சிவத்தின் சன்னிதான விசேஷத்தால் அவை சுதந்தர முடையனபோற் காணப்படுகின்றன" வென்னுமிரகசியார்த்தத்தைக் குறிப்பித்து நிற்பதாம்.

\section{சிதம்பர இரகசியமும் நடராஜ தத்துவமும்}

அந்நடராஜப்பெருமான், திருவடிநகாரமாகவும், திருவுந்திமகாரமாகவும், திருத்தோள்சிகாரமாகவும், திருமுகம்வகாரமாகவும், திருமுடியகாரமாகவும் கொண்டு, ஹிருதய குகையாகிய தகராகாச மென்னும் 
சிதம்பரத்தின்கண் ஆநந்த தாண்டவஞ் செய்தலைச் சற்குரு மூலமாய்ச் சந்தேகவிபரீதங்களற வறிந்து அந்நடனத்தின் உண்மையை யாய்ந்து அறிபவர்க்கே இனிப் பிறவா நிலையுண்டாம்.

\section{பஞ்சபூத ஸ்தலங்கள்}

பிருதிவி, அப்பு, தேயு, வாயு, ஆகாயம் என்னும் பூத பஞ்சலிங்க ஸ்தலங்களில் சிதம்பரம் ஆகாயலிங்க ஸ்தலமாயிருத்தலால் அதன்கண்ணெழுந் தருளியானந்தத் தாண்டவஞ் செய்யும் எம்பெருமானது திருக்கைக்குறி ஆகாயம் போல ஒன்றோடும் பற்றாது "என்னையார் அடைக்கின்றார்களோ அவர்களை யான் எனதென்னும் இருவகைப் பற்றினின்றும் நீக்கிப் பரமானந்தமாய் விளங்கும்படி செய்கின்றேன்" என்று அறிவிப்பது போலவாம். இதுபற்றித் தான் திருநாவுக்கரசு சிவாமிகள் "கைந்நின்ற வாடல்கண்டாற் பின்னைகண் கொண்டு காண்பதென்னே" என்று திருவாய் மலர்ந்தனர்.

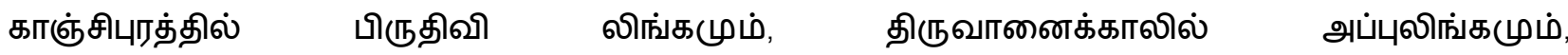
திருவண்ணாமலையில் தேயுலிங்கமும், சிகாளத்தியில் வாயுலிங்கமும், சிதம்பரத்தில் ஆகாயலிங்கமு மிருத்தலால் சிதம்பரநடனமூர்த்தி உண்மையாகத் தமக்கெனக் குறிக்கப்பட்ட ஓருருவு மில்லாதவ ரென்பதும் அன்பர்கள் விரும்பியவாறு பல்வேறு உருவங்களைக் கொள்ளுபவ ரென்பதும் அறியத்தக்கனவாம். அங்ஙனமறிந்தவர்க்கே சித்தசாந்தி சிதம்பரமாத் தானேதிகழும் அனுபவமுண்டாம்.

\section{பஞ்ச நடனசபைகள்}

இந்நடராஜப்பிரான் நடனஞ் செய்யும் ஸ்தலங்கள் சிறப்பாகவைந்தாம். அவை திருவாலங்காடு, சிதம்பரம், மதுரை, திருநெல்வேலி, திருக்குற்றாலமென்பனவாம். இவற்றுள் நடனசபைகள் முறையே இரத்தினசபை, பொற்சபை, இரசித (வெள்ளி) சபை, தாமிர (செம்பு) சபை, சித்திரசபை யென்பனவாம். இவ்வைந்து சபையிலும் அன்புடன் சென்று அருள் நடங் கண்டு இன்புருவானவரே இனிப்பிறவாநிலையை யடைவரென்க.

சிவபெருமான் அவுணர்களின் முப்புரத்தை எரித்த பொழுது ஆடிய ஆடல்கள் கொடுகொட்டி பாண்டரங்கம், கபாலம் எனச் சுட்டப்பெறுகின்றன. இவை சிவபெருமானை ஆடல்வல்லானாகக் குறிப்பிடுவதற்கான அடிப்படைகளாகும். சங்க இலக்கியத்தில் காணப்பெறும் ஆடல் பற்றிய இப்பதிவுகள் இறைத் தத்துவத்தை விளக்குவனவாக உள்ளன. ஆடலன் சிவபெருமான் அவுணர்களின் முப்புரத்தை எரித்து அந்த இடுகாட்டில் நின்று ஆடியதை,

"பொன்றார்

எயில்எரி யூட்டிய வில்லன் பயிலிருள்

காடமர்ந்து ஆடிய ஆடலன்" (பதிற்.கடவுள் வாழ்த்து:2-4)

என்பது சுட்டும். சிவபெருமான் கெடாத அவுணருடைய எயில் மூன்றும் வெந்து அழியுமாறு தீயூட்டி அழித்த வில்லையுடையவன்: செறிந்த இருளில் சுடுகாட்டை விரும்ப அவ்விடத்தே நின்று ஆடிய கூத்தன் என்பது பொருளாகும். எல்லா உயிரும் அழிந்து ஒடுங்குங்கால் இறைவன் ஒருவனே தனித்து நின்று ஆடுமிடம் சுடுகாடு. அதனைச் சுட்டுவதாகக் காடமர்;ந்து ஆடிய ஆடலன் என்பது உள்ளது.

சிவபெருமான் சுடலையில் ஆடுபவன் என்பது அனைவரும் அறிந்த ஒன்று. சுடலையை இங்கே 'இருள் பயில் காடு' என்ற தொடரால் சொல்லியிருக்கிறார்கள். அங்கேயே நிலையாக இருந்து ஆடுபவன் என்பதால் 'அமர்ந்து ஆடிய' என்றார்கள். அவன் ஆடல் வல்லான் என்பதால் 'ஆடலன்' என்றார்கள். 


\section{கொடுகொட்டி}

கொடுகொட்டி என்பது கொடுங்கொட்டி என்பதன் விகாரமாகும். தேவர்கள் வேண்டச் சிவபெருமான் முப்புரத்தை எரித்தபொழுது அவுணர் வெந்து விழுந்து வெண் சாம்பலாயினர். அவ்வாறு முப்புரம் தீப்பற்றி எரிய அதனைக் கண்டு இறையவன் இரங்காது கைகொட்டி ஆடிய ஆடல் கொடுகொட்டி எனப்பட்டது. அச்செயல் கொடுமையுடைத்தாதல் கருதி கொடுங்கொட்டி என வழங்கியது. சிவபெருமான் முப்புரத்தை எரித்து ஆடியபொழுது பறை முதலிய பல வாச்சியங்களைக் கையில் கொண்டு, பல வடிவங்களையும் தன்னிடத்தே அடக்கிக் கொண்டு ஆடினார். அப்பொழுது உமையவள் ஒருபால் நின்று சீர் என்னும் தாளத்தைச் செலுத்தினாள். சிவபெருமானின் இத்தகைய கொடுகொட்டி ஆடலை,

\section{“படுபறை இயம்பப் பல்லுருவம் பெயர்த்துநீ}

கொடுகொட்டி ஆடுங்காற் கோடுயர் அகல்குறிக்

கொடிபுரை நுகப்பினாள் கொண்டசீர் தருவாளோ” (கலித்.கடவுள் வாழ்த்து : 5-7)

என்பது உணர்த்தும். இதனை மாதவி ஆடிய பதினொரு வகை ஆடல்களுள் இளங்கோவடிகள் சுட்டுவதும் ஒப்பிடத்தக்கதாகும். கடலாடு காதையில்,

"பாரதி யாடிய பாரதி அரங்கத்துத்

திரிபுரம் எரியத் தேவர் வேண்ட

எரிமுகப் பேரம்பு ஏவல் கேட்ப

உமையவள் ஒருதிறனாக வோங்கிய

இமையவன் ஆடிய கொடுகொட்டி ஆடலும்" (சிலப்.6:39-43)

எனக் கொடுகொட்டியாடல் குறிப்பிடப்பட்டுள்ளது (வேங்கடசாமி நாட்டார், 2010). கலித்தொகை உரையில் நச்சினார்க்கினியர் கொடுகொட்டியாடலை விளக்கும் வகையில்,

“கொட்டி யாடற் கேற்ற மொட்டிய

உமையவ ளொருபாலாக வொருபால்

இமையா நாட்டத் திறைவ னாகி

அவுண நின்னுயிர் இழப்ப அக்களம்

பொலிய ஆடினன் என்ப”

என்னும் பழம்பாடலைக் காட்டுவர். இவற்றால் சிவபெருமான் முப்புரத்தை எரித்த பொழுது உமையவள் ஒரு திறனாக நிற்க ஆடிய ஆடல் கொடுகொட்டி என்பதும் இது காலப்போக்கில் ஆடல்மரபாக வளர்ந்தது என்பதும் விளங்கும். இவ்வாடல் ஆடும்பொழுது அச்சம், வியப்பு, விருப்பம், பொலிவு ஆகிய மெய்ப்பாடுகள் பொருந்த ஆடுதல் மரபாகும்.

\section{பாண்டரங்கம்}

சிவபெருமான் முப்புரத்தை எரித்து அவுணர்கசை; சாம்பலாக்கி அதனை உடம்பில் பூசி ஆடிய ஆடல் பாண்டரங்கம் எனப்படும். சிவபெருமான் அவ்வாறு ஆடும்பொழுது உமையம்மை ஒருபுறம் நின்று தூக்கு என்னும் தாளத்தைச் செலுத்தினாள். இதனை,

“மண்டமர் பலகடந்து மதுகையால் நீறணிந்து

பண்டரங்க மாடுங்காற் பனையெழில் அணைமென்றோள்

வண்டரற்றுங் கூந்தலாள் வளர்தூக்குத் தருவாளோ" (கலித்.கடவுள் வாழ்த்து: 8-10) 
என்பது உணர்த்தும். பாண்டு என்பது வெண்மை நிறத்தைச் சுட்டும். பாண்டரங்கம் என்பதற்கு வெள்ளையரங்கம் என்பது பொருளாகும். அது சாம்பல் நிறைந்த இடுகாட்டைக் குறிக்கும். சாம்பல் பூசி இடுகாட்டில் ஆடும் கூத்துக்கு அதுவே பெயராகப் பாண்டரங்கம் என ஆயிற்று. இவ்வாடலை இளங்கோவடிகள் மாதவி ஆடிய பதினொரு வகை ஆடல்களும் ஒன்றாக,

"தேர்முன் நின்ற திசைமுகன் காணப்

பாரதிஆடிய வியன்பாண் டரங்கமும்” (சிலப்.6:44-45)

எனச் சுட்டுவர். பாரதி என்பது பைரவியைக் குறிக்கும். முப்புரம் எரித்த பொழுது தேரின்முன் நின்ற நான்முகன் காணுமாறு பாரதி வடிவாய் இறைவன் வெண்ணீற்றை அணிந்து ஆடியது பாண்டரங்கம் என்று குறிப்பிடப்பட்டுள்ளது. நச்சினார்க்கினியரும் கலித்தொகை உரையில் இவ்வாடல் இயல்பை,

‘ஏறமர் கடவுண் மூவெயில் எய்வழிக்

கூறகூ றாகக் கொடியொடும் படையொடும்

வேறுவே றுருவின் விண்மிசைப் பரத்தனர்

அவ்வழி யொளியொடும் உருவொடுந் தோன்றித்

தேர்முன் நின்று திசைதலை பனிப்ப

கடிய காலக் காற்றென வேற்றவன்

படிநிலை திரியாப் பாண்டரங் கம்மே

என்ற பழம்பாடல் கொண்டு விளக்குவர். இவற்றால் சிவபெருமான் முப்புரத்தை எரித்தபொழுது வெண்ணீறணிந்து ஆடிய ஆடல் பாண்டரங்கம் என்பதும் காலப்போக்கில் ஆடல் மரபாக வளர்ந்தது என்பதும் பெறப்படும். சிலப்பதிகாரம் பாரதி வடிவாய் இறைவன் என்றும் தேர்முன்நின்ற நான்முகன் காணுமாறு ஆடியது என்றும் குறிப்பிடும் கருத்துகள் இதுபற்றிய கருத்து வளர்ச்சியைச் சுட்டுவனவாகும்.

\section{கபாலம்}

சிவபெருமான் முப்புரம் எரித்தபொழுது அவுணர்களின் தலை ஒரு முதலிய எலும்புகளைத் தாங்கி ஆடிய கூத்து கபாலக் கூத்தாகும். சிவபெருமான் அவ்வாறு ஆடிய பொழுது உமையவள் ஒருபுறம் நின்று பாணி என்னும் தாளத்தைச் செலுத்தினாள். இதனை,

“கொலையுழுவைத் தோலசைஇக் கொன்றைத்தார் சுவற்புறளத்

தலையங்கை கொண்டுநீ கபாலம் ஆடுங்கால்

முலையணிந்த முறுவலாள் முற்பாணி தருவாளோ” (கலித்.கடவுள் வாழ்த்து:11-13)

என்பது உணர்த்தும். இதில் சிவபெருமான் புலித்தோலடை உடுத்து, கொன்றை மாலை தோளில் அசையக் கபாலததை அகங்கையில் கொண்டு கபாலக் கூத்து ஆடியமை சுட்டப்பட்டுள்ளது. இளங்கோவடிகள் பதினொரு வகை ஆடல்களுள் ஒன்றாக இதனைச் சுட்டாமையும் நச்சினார்க்கினியர் இவ்வாடல் பற்றி விளக்காமையும் கொண்டு இது பிற்காலத்தில் தனி ஆடல்மரபாக வளர்ச்சி பெறவில்லை என்பது பெறப்படும்.

\section{குடக்கூத்தும் மல்லாடலும்}

குடக்கூத்தும் மல்லாடலும் திருமாலுடன் இணைத்துப் பேசப்படும் ஆடல் வகைகளாகும். திருமால் மல்லரை அழித்தபோது நிகழ்த்திய மல்லாடலைக் கலித்தொகை சுட்ட, மண்ணாலும் 
உலோகத்தாலுமான குடங்களைக் கொண்டு அவர் நிகழ்த்திய குடக்கூத்தைப் பரிபாடல் பேசுகிறது. சிவபெருமானும் விஷ்ணுவும் ஆடிய இவ்வைந்து கூத்துகளுள் நான்கினை மாதவி ஆடியதாகச் சிலப்பதிகாரம் கூறுவதால், இவற்றை அல்லது இவற்றுள் சிலவற்றைச் சங்க விறலியரும் ஆடினர் எனக் கொள்ளலாம்.

\section{ஆடல் தத்துவம்}

முப்புரமாவது மும்மலக் காரியமாகும். முப்புரத்தை எரித்தல் மும்மலத்தை எரித்தலை உணர்த்தும். எல்லா உயிரும் அழிந்து ஒடுங்குங்கால் இறைவன் ஒருவருனே தனித்து நின்று ஆடும் இடம் சுடுகாடாகும். இது இறைவன் வெண்ணீற அணிவதாலும் உணர்த்தப்பெறும். இறைவன் அழித்தற் தொழிலை நிகழ்த்துகின்ற காலங்களில் பாணியுந் தூக்கும் சீருமென்று சொலலப்பட்டவற்றை உமாதேவி காப்ப ஆடுவான். காத்தற்தொழிலை நிகழ்த்துகின்ற காலத்தில் ஒரு வடிவாய்த் திகழ்வான். இதனை,

"பாணியுந் தூக்குஞ் சீரும் என்றிவை

மாணிழை யரிவை காப்ப

வாணயில் பொருள்மைக்கு அமர்ந்தனையாடி" (கலித்.கடவுள் வாழ்த்து:15-17)

என்பது உணர்த்தும்.

\section{முடிவுரை}

சங்க இலக்கியத்தில் ஆலமர்செல்வன் குறித்த செய்திகள் அவன் போகியாய் இருந்து உயிர்க்குப் போகத்தைப் புரிதல் மட்டுமல்லாது யோகியாய் இருந்து உயிர்க்கு யோகத்தையும் அளுளுபவன் என்பதைப் புலப்படுத்துகிறது. மேலும் சிவன் அவுணர்களின் முப்புரத்தை எரித்து, கொடுகொட்டி, பாண்டரங்கம், கபாலம் ஆகிய கூத்துக்கள் ஆடியமை அவனை ஆடல்வல்லானாகக் காட்சிப்படுத்துகின்றது. இவற்றுள் கொடிகொட்டியும் பாண்டரங்கமும் பிற்காலத்தில் ஆடல் மரபாக வளர்ந்தன. இவை இறைவன் உயிர்களின் மும்மலம் எரித்தலையும் அழித்தல், காத்தல் ஆகிய தொழில்களையும் உணர்த்துவனவாகும். சிவபெருமானின் மகேசுவரத் திருமேனிகளுள் நடராசர் என்னும் ஆடல் வல்லான் தனித்துச் சுட்டப்படுதற்கு இம்மரபே அடிப்படையாகும்.

\section{References}

Puliyur Kesigan, (2010), Purananooru: Moolamum Uraiyum, Saradha pathipagam, Chennai, India

Somasundaranar, P.V., (1980), Pathupattu: Moolamum Uraiyum, South India Saiva Siddhanta Works

Publishing Society, Thirunalveli, India

Venkatasamy Nattar, N.M., (2010) Silapthikaram Moolamum Uraiyum, Ramaiah Pathippagam, Chennai, India

Funding: NIL

Acknowledgement: NIL

Conflict of Interest: NIL

About the License:

\section{(c) (1)}

Attribution 4.0 International (CC BY 4.0)
(C) The author 2021. The text of this article is licensed under a Creative Commons Attribution 4.0 International License 